\title{
Fixed Point Theorems for Nonlinear Operators with and without Monotonicity in Partially Ordered Banach Spaces
}

\author{
Hui-Sheng Ding, ${ }^{1}$ Jin Liang, ${ }^{2}$ and Ti-Jun Xiao ${ }^{3}$ \\ ${ }^{1}$ College of Mathematics and Information Science, Jiangxi Normal University, Nanchang, \\ Jiangxi 330022, China \\ ${ }^{2}$ Department of Mathematics, Shanghai Jiao Tong University, Shanghai 200240, China \\ ${ }^{3}$ Shanghai Key Laboratory for Contemporary Applied Mathematics, School of Mathematical Sciences, \\ Fudan University, Shanghai 200433, China
}

Correspondence should be addressed to Ti-Jun Xiao, xiaotj@ustc.edu.cn

Received 30 September 2009; Revised 5 December 2009; Accepted 6 December 2009

Academic Editor: Mohamed A. Khamsi

Copyright (c) 2010 Hui-Sheng Ding et al. This is an open access article distributed under the Creative Commons Attribution License, which permits unrestricted use, distribution, and reproduction in any medium, provided the original work is properly cited.

We establish two fixed point theorems for nonlinear operators on Banach spaces partially ordered by a cone. The first fixed point theorem is concerned with a class of mixed monotone operators. In the second fixed point theorem, the nonlinear operators are neither monotone nor mixed monotone. We also provide an illustrative example for our second result.

\section{Introduction}

Fixed point theorems for nonlinear operators on partially ordered Banach spaces have many applications in nonlinear equations and many other subjects (cf., e.g., [1-7] and references therein); in particular, various kinds of fixed point theorems for mixed monotone operators are proved and applied (see, e.g., $[1,3,5,7]$ and references therein).

Stimulated by $[7,8]$, we investigate further, in this paper, the existence of fixed points of nonlinear operators with and without monotonicity in partially ordered Banach spaces.

In Section 2, a fixed point theorem for a class of mixed monotone operators is established. In Section 3, without any monotonicity assumption for a class of nonlinear operators, we obtain a fixed point theorem by using Hilbert's projection metric.

Let us recall some basic notations about cone (for more details, we refer the reader to [2]). Let $X$ be a real Banach space. A closed convex set $P$ in $X$ is called a convex cone if the 
following conditions are satisfied:

(i) if $x \in P$, then $\lambda x \in P$ for any $\lambda \geq 0$,

(ii) if $x \in P$ and $-x \in P$, then $x=0$.

A cone $P$ induces a partial ordering $\leq$ in $X$ by

$$
x \leq y \quad \text { iff } y-x \in P .
$$

For any given $u, v \in P$,

$$
[u, v]:=\{x \in X \mid u \leq x \leq v\} .
$$

A cone $P$ is called normal if there exists a constant $k>0$ such that

$$
0 \leq x \leq y \text { implies that }\|x\| \leq k\|y\|,
$$

where $\|\cdot\|$ is the norm on $X$.

Throughout this paper, we denote by $\mathbb{N}$ the set of nonnegative integers, $\mathbb{R}$ the set of real numbers, $X$ a real Banach space, $P$ a convex cone in $X, e$ an element in $P \backslash\{\theta\}$ ( $\theta$ is the zero element of $X)$, and $P_{e}$ the following set:

$$
P_{e}=\{x \in P: \exists \alpha, \beta>0 \text { such that } \alpha e \leq x \leq \beta e\} .
$$

\section{Monotonic Operators}

Theorem 2.1. Suppose that the operator $A: P_{e} \times P_{e} \times P_{e} \rightarrow P_{e}$ satisfies the following.

(S1) $A(\cdot, y, z)$ is increasing, $A(x, \cdot, z)$ is decreasing, and $A(x, y, \cdot)$ is decreasing.

(S2) There exist a constant $t_{0} \in[0,1)$ and a function $\phi:(0,1) \times P_{e} \times P_{e} \rightarrow(0,+\infty)$ such that for each $x, y, z \in P_{e}$ and $t \in\left(t_{0}, 1\right), \phi(t, x, y)>t$ and

$$
A\left(t x, t^{-1} y, z\right) \geq \phi(t, x, y) A(x, y, z)
$$

(S3) There exist $x_{0}, y_{0} \in P_{e}$ such that $x_{0} \leq y_{0}, x_{0} \leq A\left(x_{0}, y_{0}, x_{0}\right), A\left(y_{0}, x_{0}, y_{0}\right) \leq y_{0}$ and

$$
\inf _{x, y \in\left[x_{0}, y_{0}\right]} \phi(t, x, y)>t, \quad \forall t \in\left(t_{0}, 1\right)
$$

(S4) There exists a constant $L>0$ such that, for all $x, y, z_{1}, z_{2} \in P_{e}$ with $z_{1} \geq z_{2}$,

$$
A\left(x, y, z_{1}\right)-A\left(x, y, z_{2}\right) \geq-L \cdot\left(z_{1}-z_{2}\right)
$$

Then $A$ has a unique fixed point $x^{*}$ in $\left[x_{0}, y_{0}\right]$, that is, $A\left(x^{*}, x^{*}, x^{*}\right)=x^{*}$. 
Proof. The proof is divided into 4 steps.

Step 1. Let $t_{1} \in\left(t_{0}, 1\right)$ and

$$
\psi(t, x, y)=\frac{\phi\left(t_{1}, x, y\right)}{t_{1}} t, \quad t \in(0,1), \quad x, y \in P_{e}
$$

For each $t \in(0,1)$, there exists a nonnegative integer $k$ such that $t_{1}^{k+1} \leq t<t_{1}^{k}$, that is, $t_{1} \leq$ $t / t_{1}^{k}<1$. Now, by (S2), we deduce, for all $x, y, z \in P_{e}$,

$$
\begin{aligned}
A\left(t x, t^{-1} y, z\right) & =A\left(\frac{t}{t_{1}^{k}} \cdot t_{1}^{k} x, \frac{t_{1}^{k}}{t} \cdot t_{1}^{-k} y, z\right) \\
& \geq \phi\left(\frac{t}{t_{1}^{k}}, t_{1}^{k} x, t_{1}^{-k} y\right) A\left(t_{1}^{k} x, t_{1}^{-k} y, z\right) \\
& \geq \frac{t}{t_{1}^{k}} A\left(t_{1}^{k} x, t_{1}^{-k} y, z\right) \\
& \geq \frac{t}{t_{1}} A\left(t_{1} x, t_{1}^{-1} y, z\right) \\
& \geq \frac{t}{t_{1}} \phi\left(t_{1}, x, y\right) A(x, y, z) \\
& =\psi(t, x, y) A(x, y, z) .
\end{aligned}
$$

Moreover, by (S3), we get

$$
\inf _{x, y \in\left[x_{0}, y_{0}\right]} \psi(t, x, y)=\frac{\inf _{x, y \in\left[x_{0}, y_{0}\right]} \phi\left(t_{1}, x, y\right)}{t_{1}} \cdot t>t, \quad \forall t \in(0,1)
$$

Hence, in the following proof, one can assume that $t_{0}=0$ in (S2) and (S3) without loss.

Step 2. Fix $x, y \in P_{e}$. Then, there exists $\alpha \in(0,1]$ such that $x, y \in\left[\alpha x_{0}, \alpha^{-1} y_{0}\right]$. Let

$$
\Psi_{x y}(z)=\frac{A(x, y, z)+L z}{1+L}, \quad z \in P_{e}
$$

Then $\Psi_{x y}$ is an operator from $P_{e}$ to $P_{e}$, and by (S4), $\Psi_{x y}$ is increasing in $P_{e}$. Combining (S1)(S3), we have

$$
A\left(x, y, \alpha x_{0}\right) \geq A\left(\alpha x_{0}, \alpha^{-1} y_{0}, x_{0}\right) \geq \phi\left(\alpha, x_{0}, y_{0}\right) A\left(x_{0}, y_{0}, x_{0}\right) \geq \alpha x_{0}
$$


provided that $\alpha \in(0,1)$. Moreover, it is easy to see that $(2.8)$ holds when $\alpha=1$. Similarly, one can show that

$$
A\left(x, y, \alpha^{-1} y_{0}\right) \leq \alpha^{-1} y_{0}
$$

Then, it follows that

$$
\Psi_{x y}\left(\alpha x_{0}\right) \geq \alpha x_{0}, \quad \Psi_{x y}\left(\alpha^{-1} y_{0}\right) \leq \alpha^{-1} y_{0}
$$

Let

$$
\begin{gathered}
x_{x y}^{n}=\Psi_{x y}\left(x_{x y}^{n-1}\right), \quad y_{x y}^{n}=\Psi_{x y}\left(y_{x y}^{n-1}\right), \quad n=1,2, \ldots, \\
x_{x y}^{0}=\alpha x_{0}, y_{x y}^{0}=\alpha^{-1} y_{0} .
\end{gathered}
$$

Then, using arguments similar to those in the proof of [7, Theorem 2.1], one can show that $\Psi_{x y}$ has a unique fixed point $x_{x y}^{*}$ in $\left[\alpha x_{0}, \alpha^{-1} y_{0}\right]$, and

$$
x_{x y}^{n} \longrightarrow x_{x y}^{*} \quad y_{x y}^{n} \longrightarrow x_{x y}^{*} \quad(n \longrightarrow \infty)
$$

We claim that $x_{x y}^{*}$ is the unique fixed point of $\Psi_{x y}$ in $P_{e}$. In fact, let $y_{x y}^{*}$ be a fixed point of $\Psi_{x y}$ in $P_{e}$, and $\beta \in(0, \alpha)$ such that $y_{x y}^{*} \in\left[\beta x_{0}, \beta^{-1} y_{0}\right]$. By the above proof, $\Psi_{x y}$ has a unique fixed point in $\left[\beta x_{0}, \beta^{-1} y_{0}\right]$, which means that $x_{x y}^{*}=y_{x y}^{*}$. In addition, it follows from

$$
x_{x y}^{*}=\Psi_{x y}\left(x_{x y}^{*}\right)=\frac{A\left(x, y, x_{x y}^{*}\right)+L x_{x y}^{*}}{1+L}
$$

that $x_{x y}^{*}=A\left(x, y, x_{x y}^{*}\right)$.

Step 3. By Step 2, we can define an operator $\Phi: P_{e} \times P_{e} \rightarrow P_{e}$ by

$$
\Phi(x, y)=x_{x y}^{*}=\Psi_{x y}\left(x_{x y}^{*}\right)=A\left(x, y, x_{x y}^{*}\right) .
$$

Let $x, x^{\prime} \in\left[x_{0}, y_{0}\right]$ with $x \leq x^{\prime}$ and $\alpha \in(0,1]$ with $x, x^{\prime}, y \in\left[\alpha x_{0}, \alpha^{-1} y_{0}\right]$. Denote by $\left\{x_{x y}^{n}\right\},\left\{x_{x^{\prime} y}^{n}\right\}$ the corresponding sequences in the proof of Step 2. Then

$$
\begin{aligned}
x_{x y}^{1} & =\Psi_{x y}\left(x_{x y}^{0}\right)=\Psi_{x y}\left(\alpha x_{0}\right)=\frac{A\left(x, y, \alpha x_{0}\right)+L \alpha x_{0}}{1+L} \\
& \leq \frac{A\left(x^{\prime}, y, \alpha x_{0}\right)+L \alpha x_{0}}{1+L}=\Psi_{x^{\prime} y}\left(\alpha x_{0}\right)=x_{x^{\prime} y}^{1} .
\end{aligned}
$$


Next, by induction and $\Psi_{x y}$ being increasing, one can show that $x_{x y}^{n} \leq x_{x^{\prime} y}^{n}$ for all $n \in \mathbb{N}$. So

$$
x_{x y}^{*}=\lim _{n \rightarrow \infty} x_{x y}^{n} \leq \lim _{n \rightarrow \infty} x_{x^{\prime} y}^{n}=x_{x^{\prime} y^{\prime}}^{*}
$$

that is, $\Phi(x, y) \leq \Phi\left(x^{\prime}, y\right)$. Thus, $\Phi(\cdot, y)$ is increasing. By a similar method, one can prove that $\Phi(x, \cdot)$ is decreasing. On the other hand, by (S3), for $x, y \in P_{e}$ and $t \in(0,1)$,

$$
\begin{aligned}
\Phi\left(t x, t^{-1} y\right) & =A\left(t x, t^{-1} y, \Phi\left(t x, t^{-1} y\right)\right) \\
& \geq A\left(t x, t^{-1} y, \Phi(x, y)\right) \\
& \geq \phi(t, x, y) A(x, y, \Phi(x, y)) \\
& =\phi(t, x, y) \Phi(x, y) .
\end{aligned}
$$

Let $u_{0}=x_{0}, v_{0}=y_{0}$, and

$$
u_{n}=\Phi\left(u_{n-1}, v_{n-1}\right), \quad v_{n}=\Phi\left(v_{n-1}, u_{n-1}\right), \quad \text { for } n=1,2, \ldots
$$

By choosing $\alpha=1$ in Step 1 , we get $x_{x_{0} y_{0}}^{*} \in\left[x_{0}, y_{0}\right]$. Then

$$
u_{1}=\Phi\left(x_{0}, y_{0}\right)=x_{x_{0} y_{0}}^{*} \geq x_{0}=u_{0}, \quad v_{1}=\Phi\left(y_{0}, x_{0}\right)=x_{y_{0} x_{0}}^{*} \leq y_{0}=v_{0}
$$

As $\Phi(\cdot, y)$ is increasing and $\Phi(x, \cdot)$ is decreasing, it follows immediately that

$$
u_{0} \leq u_{1} \leq \cdots \leq u_{n} \leq \cdots \leq v_{n} \leq \cdots \leq v_{0} .
$$

Next, by making some needed modifications in the proof of [3, Theorem 2.11], one can show that $\Phi$ has a fixed point $x^{*} \in\left[x_{0}, y_{0}\right]$. Suppose that $y^{*} \in\left[x_{0}, y_{0}\right]$ is a fixed point of $\Phi$. It follows from the definition of $u_{n}$ and $v_{n}$ that $u_{n} \leq y^{*} \leq v_{n}$ for all $n \in \mathbb{N}$. Then, by the normality of $\Phi$, we get $y^{*}=x^{*}$. So $x^{*}$ is the unique fixed point of $\Phi$ in $\left[x_{0}, y_{0}\right]$.

Step 4. By Steps 2 and 3, we get

$$
x^{*}=\Phi\left(x^{*}, x^{*}\right)=A\left(x^{*}, x^{*}, \Phi\left(x^{*}, x^{*}\right)\right)=A\left(x^{*}, x^{*}, x^{*}\right) .
$$

Let $\bar{x} \in\left[x_{0}, y_{0}\right]$ such that $\bar{x}=A(\bar{x}, \bar{x}, \bar{x})$. Then it follows from Step 2 that $\Phi(\bar{x}, \bar{x})=\bar{x}$, that is, $\bar{x}$ is a fixed point of $\Phi$ in $\left[x_{0}, y_{0}\right]$. Thus, by Step $3, \bar{x}=x^{*}$, which means that $x^{*}$ is the unique fixed point of $A$ in $\left[x_{0}, y_{0}\right]$.

Remark 2.2. Compared with [7, Remark 2.4], the nonlinear operator $A$ in Theorem 2.1 is more general, and so Theorem 2.1 may have a wider range of applications. 


\section{Nonmonotonic Case}

First, let us recall some definitions and basic results about Hilbert's projection metric (for more details, see [6]).

Definition 3.1. Elements $x$ and $y$ belonging to $P$ (not both zero) are said to be linked if there exist $\lambda, \mu>0$ such that

$$
\lambda x \leq y \leq \mu x .
$$

This defines an equivalence relation on $P$ and divides $P$ into disjoint subsets which we call constituents of $P$.

Let $x$ and $y$ be linked. Define

$$
\begin{aligned}
M(x, y) & =\inf \{\mu>0: y \leq \mu x\} \\
d(x, y) & =\ln [\max \{M(x, y), M(y, x)\}] .
\end{aligned}
$$

Then, the following holds.

Theorem 3.2. $d(\cdot, \cdot)$ defines a complete metric on each constituent of $P$.

Proof. See [6].

We will also need the following result.

Theorem 3.3. [9] Let $M$ be a complete metric space and suppose that $f: M \rightarrow M$ satisfies

$$
d(f(x), f(y)) \leq \Psi(d(x, y)), \quad \forall x, y \in M,
$$

where $\Psi:[0,+\infty) \rightarrow[0,+\infty)$ is upper semicontinuous from the right and satisfies $\Psi(t)<t$ for all $t>0$. Then $f$ has a unique fixed point in $M$.

Theorem 3.3 is a generalization of the classical Banach's contraction mapping principle. There are many generalizations of the classical Banach's contraction mapping principle (see, e.g., [10, 11] and references therein), and these generalizations play an important role in research work about fixed points of nonlinear operators in partially ordered Banach spaces; see, for example, [1] and the proof of the following theorem.

Now, we are ready to present our fixed point theorem, in which no monotone condition is assumed on the nonlinear operator.

Theorem 3.4. Let $T$ be an operator from $P_{e}$ to $P_{e}$. Assume that there exist a constant $\varepsilon \in(0,1)$ and $a$ function $\phi:[\varepsilon, 1) \rightarrow(0,+\infty)$ such that $\phi(\lambda)>\lambda$ for all $\lambda \in[\varepsilon, 1)$, and

$$
T y \geq \phi(\lambda) T x,
$$

for all $x, y \in P_{e}$ and $\lambda \in[\varepsilon, 1)$ satisfying $\lambda x \leq y \leq \lambda^{-1} x$. Then $T$ has a unique fixed point in $P_{e}$. 
Fixed Point Theory and Applications

Proof. We divided the proof into 2 steps.

Step 1. Let $\lambda \in(0,1), x, y \in P_{e}$, and $\lambda x \leq y \leq \lambda^{-1} x$. Then, there exists $k \in \mathbb{N}$ such that

$$
\varepsilon \leq \frac{\lambda}{\varepsilon^{k}}<1
$$

In view of

$$
\frac{\lambda}{\varepsilon^{k}} \cdot\left(\varepsilon^{k} x\right)=\lambda x \leq y \leq \lambda^{-1} x \leq \varepsilon^{2 k} \lambda^{-1} x=\left(\frac{\lambda}{\varepsilon^{k}}\right)^{-1} \cdot\left(\varepsilon^{k} x\right),
$$

by the assumptions, we have

$$
T y \geq \phi\left(\frac{\lambda}{\varepsilon^{k}}\right) \cdot T\left(\varepsilon^{k} x\right) \geq \frac{\lambda}{\varepsilon^{k}} \cdot T\left(\varepsilon^{k} x\right) .
$$

Similar to the above proof, since $\varepsilon \cdot \varepsilon^{k-1} x=\varepsilon^{k} x \leq \varepsilon^{-1} \cdot \varepsilon^{k-1} x$, one can deduce

$$
T y \geq \frac{\lambda}{\varepsilon^{k}} \cdot T\left(\varepsilon^{k} x\right) \geq \frac{\lambda}{\varepsilon^{k}} \cdot \phi(\varepsilon) \cdot T\left(\varepsilon^{k-1} x\right) \geq \frac{\lambda}{\varepsilon^{k-1}} \cdot T\left(\varepsilon^{k-1} x\right) .
$$

Continuing by this way, one can get

$$
T y \geq \frac{\lambda}{\varepsilon} \cdot T(\varepsilon x) \geq \frac{\phi(\varepsilon)}{\varepsilon} \lambda \cdot T x
$$

Let

$$
\psi(\lambda)=\frac{\phi(\varepsilon)}{\varepsilon} \lambda, \quad \lambda \in(0,1)
$$

Then $\psi$ is continuous, $\psi(\lambda)>\lambda$ for all $\lambda \in(0,1)$, and

$$
T y \geq \psi(\lambda) T x,
$$

for all $x, y \in P_{e}$ and $\lambda \in(0,1)$ satisfying $\lambda x \leq y \leq \lambda^{-1} x$.

Step 2. Next, let $x, y \in P_{e}$ with $x \neq y$ and

$$
\lambda=\frac{1}{\max \{M(x, y), M(y, x)\}} .
$$

Then $\lambda \in(0,1), \lambda x \leq y \leq \lambda^{-1} x$, and $d(x, y)=\ln \left(\lambda^{-1}\right)$. Moreover, by Step 1, we have

$$
T y \geq \psi(\lambda) T x .
$$


On the other hand, since $\lambda y \leq x \leq \lambda^{-1} y$, we also have

$$
T x \geq \psi(\lambda) T y .
$$

Thus, we get

$$
\psi(\lambda) T x \leq T y \leq \frac{T x}{\psi(\lambda)} .
$$

Now, by the definition of $d(\cdot, \cdot)$, we have

$$
d(T x, T y) \leq \ln \left(\frac{1}{\psi(\lambda)}\right)
$$

Let

$$
\Psi(t)= \begin{cases}-\ln \left[\psi\left(e^{-t}\right)\right], & t \in(0,+\infty), \\ 0, & t=0 .\end{cases}
$$

Then, $\Psi$ is a continuous function from $[0,+\infty)$ to $[0,+\infty)$, and

$$
d(T x, T y) \leq \Psi(d(x, y))
$$

Moreover, since $\psi(\lambda)>\lambda$ for all $\lambda \in(0,1)$, we get

$$
\Psi(t)=\ln \frac{1}{\psi\left(e^{-t}\right)}<\ln \frac{1}{e^{-t}}=t, \quad t>0
$$

On the other hand, $P_{e}$ is obviously a constituent of $P$, and thus $\left(P_{e}, d\right)$ is complete by Theorem 3.2. Now, Theorem 3.3 yields that $T$ has a unique fixed point in $P_{e}$.

Corollary 3.5. Assume that $A: P_{e} \times P_{e} \rightarrow P_{e}$ is a mixed monotone operator, that is, $A(\cdot, y)$ is increasing and $A(x, \cdot)$ is decreasing. Moreover, there exist a constant $\varepsilon \in(0,1)$ and a function $\phi$ : $[\varepsilon, 1) \rightarrow(0,+\infty)$ such that $\phi(\lambda)>\lambda$ for all $\lambda \in[\varepsilon, 1)$, and

$$
A\left(\lambda x, \lambda^{-1} y\right) \geq \phi(\lambda) A(x, y)
$$

for all $x, y \in P_{e}$ and $\lambda \in[\varepsilon, 1)$. Then $A$ has a unique fixed point in $P_{e}$.

Proof. Let $T z=A(z, z), z \in P_{e}$. Then, since $A$ is a mixed monotone operator, we have

$$
T y=A(y, y) \geq A\left(\lambda x, \lambda^{-1} x\right) \geq \phi(\lambda) A(x, x)=\phi(\lambda) T x
$$


for all $x, y \in P_{e}$ and $\lambda \in[\varepsilon, 1)$ satisfying $\lambda x \leq y \leq \lambda^{-1} x$. Then, Theorem 3.4 yields the conclusion.

Remark 3.6. Corollary 3.5 is an improvement of [1, Corollary 3.2] in the sense that there $\phi$ is lower semicontinuous on $(0,1)$, and the corresponding conditions need to hold on the whole interval $(0,1)$.

\section{An Example}

In this section, we give an example to illustrate Theorem 3.4. Let us consider the following nonlinear delay integral equation:

$$
x(t)=\int_{t-\tau}^{t} f(s, x(s)) d s,
$$

which is a classical model for the spread of some infectious disease (cf. [12]). In fact, (4.1) has been of great interest for many authors (see, e.g., $[3,8]$ and references therein).

In the rest of this paper, let $\tau=1$ and

$$
f(t, x)=\left\{\begin{array}{lll}
\left(1+\sin ^{2} t+\sin ^{2} \pi t\right) \sqrt{x}, & t \in(-\infty,+\infty), & 0 \leq x \leq 1, \\
\frac{1+\sin ^{2} t+\sin ^{2} \pi t}{\sqrt{x}}, & t \in(-\infty,+\infty), & x \geq 1 .
\end{array}\right.
$$

Next, let us investigate the existence of positive almost periodic solution to (4.1). For the reader's convenience, we recall some definitions and basic results about almost periodic functions (for more details, see [13]).

Definition 4.1. A continuous function $f: \mathbb{R} \rightarrow \mathbb{R}$ is called almost periodic if for each $\varepsilon>0$ there exists $l(\varepsilon)>0$ such that every interval $I$ of length $l(\varepsilon)$ contains a number $\tau$ with the property that

$$
\|f(t+\tau)-f(t)\|<\varepsilon \quad \forall t \in \mathbb{R} .
$$

Denote by $A P(\mathbb{R})$ the set of all such functions.

Lemma 4.2. Assume that $f, g \in A P(\mathbb{R})$. Then the following hold.

(a) The range $\mathcal{R}_{f}=\{f(t): t \in \mathbb{R}\}$ is precompact in $\mathbb{R}$, and so $f$ is bounded.

(b) $F(f) \in A P(\mathbb{R})$ provided that $F$ is continuous on $\overline{\mathcal{R}_{f}}$.

(c) $f+g, f \cdot g \in A P(\mathbb{R})$. Moreover, $f / g \in A P(\mathbb{R})$ provided that $\inf _{t \in \mathbb{R}}|g(t)|>0$.

(d) Equipped with the sup norm

$$
\|f\|=\sup _{t \in \mathbb{R}}|f(t)|
$$

$A P(\mathbb{R})$ turns out to be a Banach space. 
Now, let $P=\{x \in A P(\mathbb{R}): x(t) \geq 0, \forall t \in \mathbb{R}\}$, and $e \in P$ is defined by $e(t) \equiv 1$. It is not difficult to verify that $P$ is a normal cone in $A P(\mathbb{R})$, and

$$
P_{e}=\{x \in A P(\mathbb{R}): \exists \varepsilon>0 \text { such that } x(t)>\varepsilon, \quad \forall t \in \mathbb{R}\} .
$$

Define a nonlinear operator $T$ on $P_{e}$ by

$$
(T x)(t)=\int_{t-1}^{t} f(s, x(s)) d s, \quad x \in P_{e}, \quad t \in \mathbb{R} .
$$

By Lemma 4.2 and [3, Corollary 3.3], it is not difficult to verify that $T$ is an operator from $P_{e}$ to $P_{e}$. In addition, in view of (4.2), one can verify that

$$
(T y)(t)=\int_{t-1}^{t} f(s, y(s)) d s \geq \sqrt{\lambda} \int_{t-1}^{t} f(s, x(s)) d s=\sqrt{\lambda}(T x)(t), \quad t \in \mathbb{R},
$$

that is, $T y \geq \sqrt{\lambda} T x$ for all $x, y \in P_{e}$ and $\lambda \in(0,1)$ with $\lambda x \leq y \leq \lambda^{-1} x$. Then, by Theorem 3.4, $T$ has a unique fixed point in $P_{e}$, that is, (4.1) has a unique almost periodic solution with positive infimum.

\section{Acknowledgments}

The authors are very grateful to the referees for valuable suggestions and comments. In addition, Hui-Sheng Ding acknowledges support from the NSF of China (10826066), the NSF of Jiangxi Province of China (2008GQS0057), and the Youth Foundation of Jiangxi Provincial Education Department(GJJ09456); Jin Liang and Ti-Jun Xiao acknowledge support from the NSF of China (10771202), the Research Fund for Shanghai Key Laboratory for Contemporary Applied Mathematics (08DZ2271900), and the Specialized Research Fund for the Doctoral Program of Higher Education of China (2007035805).

\section{References}

[1] Y. Z. Chen, "Thompson's metric and mixed monotone operators," Journal of Mathematical Analysis and Applications, vol. 177, no. 1, pp. 31-37, 1993.

[2] K. Deimling, Nonlinear Functional Analysis, Springer, Berlin, Germany, 1985.

[3] H.-S. Ding, T.-J. Xiao, and J. Liang, "Existence of positive almost automorphic solutions to nonlinear delay integral equations," Nonlinear Analysis: Theory, Methods \& Applications, vol. 70, no. 6, pp. 22162231, 2009.

[4] J. A. Gatica and W. A. Kirk, "A fixed point theorem for $k$-set-contractions defined in a cone," Pacific Journal of Mathematics, vol. 53, pp. 131-136, 1974.

[5] K. Li, J. Liang, and T.-J. Xiao, "New existence and uniqueness theorems of positive fixed points for mixed monotone operators with perturbation," Journal of Mathematical Analysis and Applications, vol. 328, no. 2, pp. 753-766, 2007.

[6] A. C. Thompson, "On certain contraction mappings in a partially ordered vector space," Proceedings of the American Mathematical Society, vol. 14, pp. 438-443, 1963.

[7] Z. Zhang and K. Wang, "On fixed point theorems of mixed monotone operators and applications," Nonlinear Analysis: Theory, Methods E Applications, vol. 70, no. 9, pp. 3279-3284, 2009. 
[8] E. Ait Dads, P. Cieutat, and L. Lhachimi, "Positive pseudo almost periodic solutions for some nonlinear infinite delay integral equations," Mathematical and Computer Modelling, vol. 49, no. 3-4, pp. 721-739, 2009.

[9] D. W. Boyd and J. S. W. Wong, "On nonlinear contractions," Proceedings of the American Mathematical Society, vol. 20, pp. 458-464, 1969.

[10] W. A. Kirk, "Fixed points of asymptotic contractions," Journal of Mathematical Analysis and Applications, vol. 277, no. 2, pp. 645-650, 2003.

[11] W. A. Kirk and H.-K. Xu, "Asymptotic pointwise contractions," Nonlinear Analysis: Theory, Methods $\mathcal{E}$ Applications, vol. 69, no. 12, pp. 4706-4712, 2008.

[12] K. L. Cooke and J. L. Kaplan, "A periodicity threshold theorem for epidemics and population growth," Mathematical Biosciences, vol. 31, no. 1-2, pp. 87-104, 1976.

[13] A. M. Fink, Almost Periodic Differential Equations, vol. 377 of Lecture Notes in Mathematics, Springer, Berlin, Germany, 1974. 